NISSUNA UMANA INVESTIGAZIONE SI PUO DIMANDARE VERA SCIENZIA S'ESSA NON PASSA PER LE MATEMATICHE DIMOSTRAZIONI LEONARDO DA VINCI

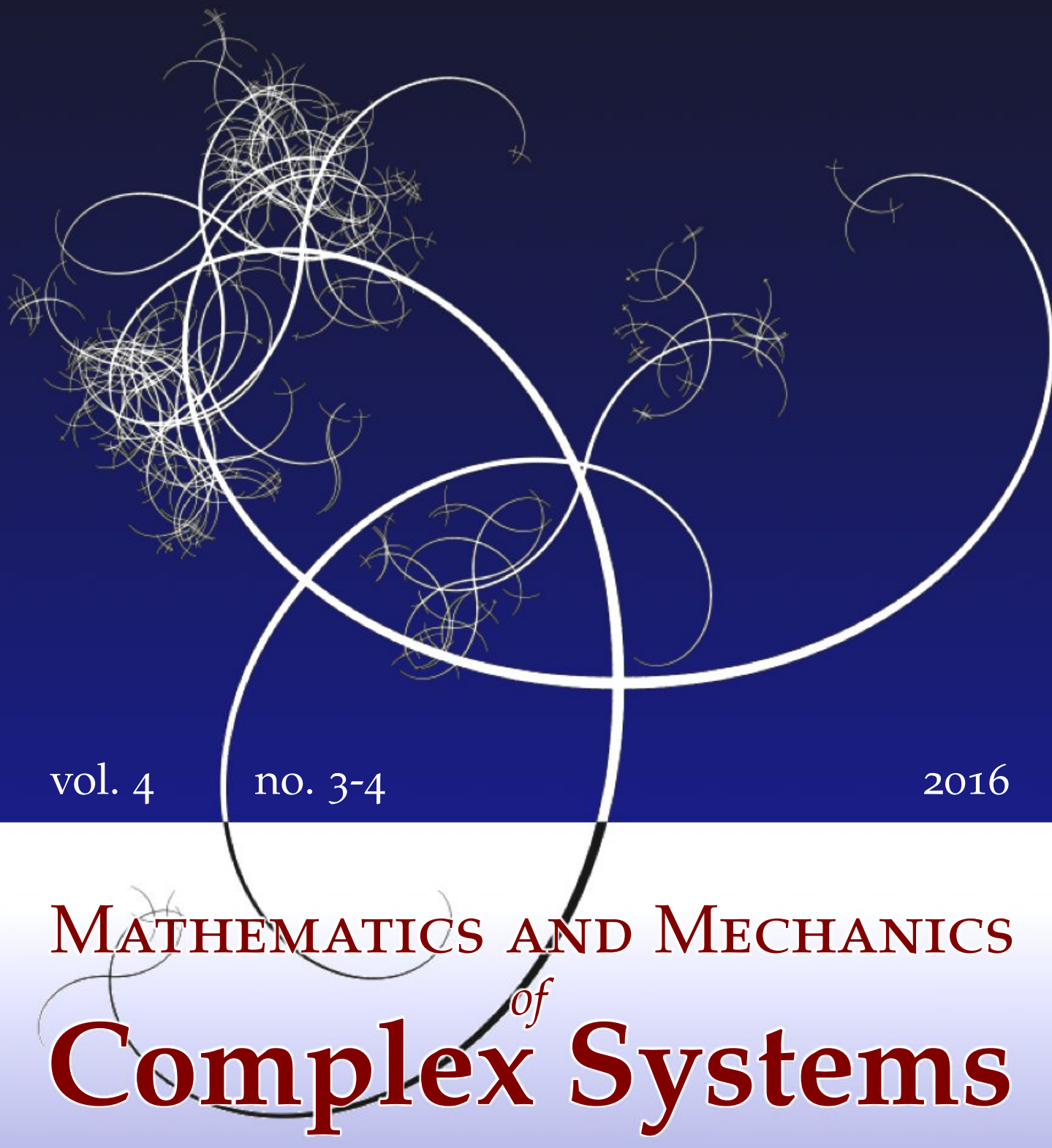

GEOFFREY R. GRIMMETT

CORRELATION INEQUALITIES FOR THE POTTS MODEL 


\title{
CORRELATION INEQUALITIES FOR THE POTTS MODEL
}

\author{
GEOFFREY R. GRIMMETT \\ Dedicated in friendship to Lucio Russo
}

\begin{abstract}
Correlation inequalities are presented for ferromagnetic Potts models with external field, using the random-cluster representation of Fortuin and Kasteleyn, together with the FKG inequality. These results extend and simplify earlier inequalities of Ganikhodjaev and Razak, and also of Schonmann, and include GKS-type inequalities when the spin space is taken as the set of $q$-th roots of unity.
\end{abstract}

\section{Introduction}

Correlation inequalities are key to the classical theory of interacting systems in statistical mechanics. The Ising model, especially, has a plethora of associated inequalities that have played significant roles in the development of a coherent theory of phase transition (see, for example, the books [7; 22]). These inequalities are frequently named after their discoverers, and include inequalities of Griffiths [14; 15; 16], Griffiths, Kelly, and Sherman (GKS) [20], Griffiths, Hurst, and Sherman (GHS) [17], Ginibre [13], Simon and Lieb [21; 24], and so on.

A more probabilistic theory of Ising/Potts models has emerged since around 1970 , initiated partly by the work of Fortuin and Kasteleyn $[8 ; 9 ; 10]$ on the random-cluster representation of the Potts model and the random-current method championed by Aizenman [1] and co-authors. Probably the principle inequality in the probabilistic formulation is that of Fortuin, Kasteleyn, and Ginibre (FKG) [11].

Inequalities are rarer for the Potts model, and our purpose in this note is to derive certain correlation inequalities for a ferromagnetic Potts model with external field, akin to the GKS inequalities for the Ising model. The main technique used here is the random-cluster representation of this model and particularly the FKG inequality.

Our results generalize and simplify the work of Ganikhodjaev and Razak [12], who have shown how to formulate and prove GKS-type inequalities for the Potts

\section{Communicated by Raffaele Esposito.}

MSC2010: 82B20, 60K35.

Keywords: Griffiths inequality, GKS inequality, Ising model, Potts model, random-cluster model, angular spins. 
model with a general number $q$ of local states. Furthermore, our Theorems 3.5 and 3.7 extend the two correlation inequalities of Schonmann [23], which in turn extended inequalities of [6]. Some of the arguments given here may be known to others.

The structure of this paper is as follows. The Potts and random-cluster models are introduced in Section 2, and the results of the paper (Theorems 3.5-3.7) follow in Section 3. The proofs are given in Sections 4, 5, and 6.

\section{The Potts model with external field}

Let $G=(V, E)$ be a finite graph, and let $J=\left(J_{e}: e \in E\right)$ and $h=\left(h_{v}: v \in V\right)$ be vectors of nonnegative reals and $q \in\{2,3, \ldots\}$. An edge $e \in E$ joins two distinct vertices $x$ and $y$, and we write $e=\langle x, y\rangle$.

We take the "local state space" for the $q$-state Potts model to be the set $\mathcal{Q}:=$ $\{0,1, \ldots, q-1\}$ of "spins". The configuration space of the model is the product space $\Sigma:=\mathcal{Q}^{V}$, and a typical configuration is written $\sigma=\left(\sigma_{v}: v \in V\right) \in \Sigma$. The Potts measure on $G$ with parameters $J$ and $h$ has sample space $\Sigma$ and probability measure given by

$$
\pi(\sigma)=\frac{1}{Z} \exp \left\{\sum_{e=\langle x, y\rangle \in E} J_{e} \delta_{e}(\sigma)+\sum_{v \in V} h_{v} \delta_{v}(\sigma)\right\}, \quad \sigma \in \Sigma,
$$

where $\delta_{e}(\sigma)=\delta_{\sigma_{x}, \sigma_{y}}$ and $\delta_{v}(\sigma)=\delta_{\sigma_{v}, 0}$ are Kronecker delta functions and $Z$ is the appropriate normalizing constant. Thus, the $J_{e}$ are edge-coupling constants, and the $h_{v}$ are external fields relative to the local state 0 . The Potts measure is said to be ferromagnetic since $J_{e} \geq 0$ for $e \in E$.

We shall make use of the random-cluster representation, for a recent account and bibliography of which we refer the reader to [18]. The graph $G$ is augmented by adding a "ghost" vertex $g$, which is joined by edges $\langle g, v\rangle$ to each vertex $v \in V$; the ensuing graph is denoted $G^{+}=\left(V^{+}, E^{+}\right)$. The relevant sample space is the product space $\Omega:=\{0,1\}^{E^{+}}$. For $\omega=\left(\omega_{e}: e \in E^{+}\right) \in \Omega$, an edge $e$ is called open if $\omega_{e}=1$ and closed otherwise.

An edge $e \in E$ is assigned parameter $p_{e}=1-e^{-J_{e}}$, and an edge of the form $\langle g, v\rangle$ is assigned parameter $p_{v}=1-e^{-h_{v}}$. The random-cluster probability measure $\phi$ on $G$ has sample space $\Omega$ and is given by

$$
\phi(\omega)=\frac{1}{Z_{\mathrm{RC}}}\left\{\prod_{e=\langle x, y\rangle \in E^{+}} p_{e}^{\omega_{e}}\left(1-p_{e}\right)^{1-\omega_{e}}\right\} q^{k(\omega)}, \quad \omega \in \Omega,
$$

where $k(\omega)$ is the number of connected components of the graph with vertex set $V^{+}$ and edge set $\eta(\omega):=\left\{e \in E^{+}: \omega_{e}=1\right\}$. 
The relationship between the Potts model and the random-cluster model is explained in [18, §1.4], where it is shown in particular that $Z_{\mathrm{RC}}=e^{-|E|} Z$.

The measures $\pi$ and $\phi$ may be coupled as follows. Suppose $\omega$ is sampled from $\Omega$ according to $\phi$, and let $C_{v}$ be the connected component of $(V, \eta(\omega))$ containing $v \in V^{+}$; the $C_{v}$ are called open clusters. Every vertex in $C_{g}$ is allocated spin 0 . To an open cluster of $\omega$ other than $C_{g}$, we allocate a uniformly chosen spin from $\mathcal{Q}$ such that every vertex in the cluster receives this spin and the spins of different clusters are independent. The ensuing spin vector $\sigma=\sigma(\omega)$ has law $\pi$. See [18, Theorem 1.3] for a proof of this standard fact and for references to the original work of Fortuin and Kasteleyn.

This paper will make use of the FKG inequality and the comparison inequalities for the random-cluster model. These are presented in a number of places already and are not repeated here. The reader is referred instead to [18, Theorem 3.8] for the FKG inequality and to [18, Theorem 3.21$]$ for the comparison inequalities.

\section{The correlation inequalities}

We begin with a space of functions. Let $\mathcal{F}_{q}$ be the set of functions $f: \mathcal{Q} \rightarrow \mathbb{C}$ such that, for all integers $m, n \geq 0$,

$$
\begin{aligned}
& \mathbb{E}\left(f(X)^{m}\right) \text { is real and nonnegative, } \\
& \mathbb{E}\left(f(X)^{m+n}\right) \geq \mathbb{E}\left(f(X)^{m}\right) \mathbb{E}\left(f(X)^{n}\right),
\end{aligned}
$$

where $X$ is a uniformly distributed random variable on $\mathcal{Q}$. The above conditions may be written out as follows. We have that $f \in \mathcal{F}_{q}$ if, for $m, n \geq 0$,

$$
\begin{aligned}
& S_{m}:=\sum_{x \in \mathcal{Q}} f(x)^{m} \text { is real and nonnegative, } \\
& q S_{m+n} \geq S_{m} S_{n} .
\end{aligned}
$$

For $I \in \mathcal{Q}$, let $\mathcal{F}_{q}^{I}$ be the subset of $\mathcal{F}_{q}$ containing all $f$ such that

$$
f(I)=\max \{|f(x)|: x \in \mathcal{Q}\} .
$$

This condition entails that $f(I)$ is real and nonnegative.

Let $f: \mathcal{Q} \rightarrow \mathbb{C}$. For $\sigma \in \Sigma$, let

$$
f(\sigma)^{R}:=\prod_{v \in R} f\left(\sigma_{v}\right), \quad R \subseteq V .
$$

Thinking of $\sigma$ as a random vector with law $\pi$, we write $\left\langle f(\sigma)^{R}\right\rangle$ for the mean value of $f(\sigma)^{R}$. 
Theorem 3.5. Let $f \in \mathcal{F}_{q}^{0}$. For $R \subseteq V$, the mean $\left\langle f(\sigma)^{R}\right\rangle$ is real-valued and nondecreasing in the vectors $J$ and $h$ and satisfies $\left\langle f(\sigma)^{R}\right\rangle \geq 0$. For $R, S \subseteq V$, we have

$$
\left\langle f(\sigma)^{R} f(\sigma)^{S}\right\rangle \geq\left\langle f(\sigma)^{R}\right\rangle\left\langle f(\sigma)^{S}\right\rangle .
$$

If there is no external field, in that $h \equiv 0$, it suffices for the above that $f \in \mathcal{F}_{q}$ in place of $f \in \mathcal{F}_{q}^{0}$.

Here are three classes of functions belonging to $\mathcal{F}_{q}^{0}$.

Theorem 3.6. Let $q \geq 2$. The following functions $f: \mathcal{Q} \rightarrow \mathbb{C}$ belong to $\mathcal{F}_{q}^{0}$ :

(a) $f(x)=\frac{1}{2}(q-1)-x$,

(b) $f(x)=e^{2 \pi i x / q}$, a $q$-th root of unity, and

(c) $f: \mathcal{Q} \rightarrow[0, \infty)$, with $f(x) \leq f(0)$ for $x \in \mathcal{Q}$.

When combined with Theorem 3.5, case (a) yields the inequalities of Ganikhodjaev and Razak [12], but with simpler proofs. When $q=2$, the latter reduce to the GKS inequalities for the Ising model; see $[14 ; 15 ; 16 ; 20]$. We do not know if the implications of Theorem 3.5 with case (b) are either known or useful. Perhaps they are examples of the results of Ginibre [13]. In case (c) with $f(x)=\delta_{x, 0}$, Theorem 3.5 yields the first correlation inequality of Schonmann [23].

Our second main result follows next.

Theorem 3.7. Let $q \geq 2$ and $f_{0} \in \mathcal{F}_{q}^{0}$, and let $f_{1}: \mathcal{Q} \rightarrow \mathbb{C}$ satisfy (3.1). If $f_{0}$ and $f_{1}$ have disjoint support in that $f_{0} f_{1} \equiv 0$, then for $R, S \subseteq V$,

$$
\left\langle f_{0}(\sigma)^{R} f_{1}(\sigma)^{S}\right\rangle \leq\left\langle f_{0}(\sigma)^{R}\right\rangle\left\langle f_{1}(\sigma)^{S}\right\rangle .
$$

If $h \equiv 0$, it is enough to assume $f_{0} \in \mathcal{F}_{q}$ in place of $f_{0} \in \mathcal{F}_{q}^{0}$.

Two correlation inequalities were proved in [23]: a "positive" inequality that is implied by Theorems 3.5 and 3.6(c) and a "negative" inequality that is obtained as a special case of Theorem 3.7 on setting $f_{0}(x)=\delta_{x, 0}$ and $f_{1}(x)=\delta_{x, 1}$. Recall that Schonmann's inequalities were themselves (partial) generalizations of correlation inequalities of [6].

Amongst the feasible extensions of the above theorems that come to mind, we mention the classical space-time models used to study the quantum Ising/Potts models $[2 ; 3 ; 4 ; 5 ; 19]$.

\section{Proof of Theorem 3.5}

We use the coupling of the random-cluster and Potts model described in Section 2. Let $\omega \in \Omega$, and let $A_{g}, A_{1}, A_{2}, \ldots, A_{k}$ be the vertex sets of the open clusters of $\omega$, where $A_{g}$ is that of the open cluster $C_{g}$ containing $g$. 
Let $R \subseteq V$, and let $f \in \mathcal{F}_{q}^{0}$. By (3.4),

$$
f(\sigma)^{R}=f(0)^{\left|R \cap A_{g}\right|} \prod_{r=1}^{k} f\left(X_{r}\right)^{\left|R \cap A_{r}\right|},
$$

where $X_{r}$ is the random spin assigned to $A_{r}$. This has conditional expectation $g_{R}: \Omega \rightarrow \mathbb{C}$ given by

$$
\begin{aligned}
g_{R}(\omega) & :=\mathbb{E}\left(f(\sigma)^{R} \mid \omega\right) \\
& =f(0)^{\left|R \cap A_{g}\right|} \prod_{r=1}^{k} \mathbb{E}\left(f(X)^{\left|R \cap A_{r}\right|} \mid \omega\right) .
\end{aligned}
$$

By (3.1) and (3.3), $g_{R}(\omega)$ is real and nonnegative, whence so is its mean $\phi\left(g_{R}\right)=$ $\left\langle f(\sigma)^{R}\right\rangle$. (It will be convenient to use $\phi(Y)$ to denote the expectation of a random variable $Y: \Omega \rightarrow \mathbb{R}$.)

We show next that $g_{R}$ is a nondecreasing function on the partially ordered set $\Omega$. It suffices to consider the case when the configuration $\omega^{\prime}$ is obtained from $\omega$ by adding an edge between two clusters of $\omega$. In this case, by (3.2) and (3.3), $g_{R}\left(\omega^{\prime}\right) \geq g_{R}(\omega)$. That $\left\langle f(\sigma)^{R}\right\rangle=\phi\left(g_{R}\right)$ is nondecreasing in $J$ and $h$ follows by the appropriate comparison inequality for the random-cluster measure $\phi[18$, Theorem 3.21].

Now,

$$
\mathbb{E}\left(f(\sigma)^{R} f(\sigma)^{S} \mid \omega\right)=f(0)^{\left|R \cap A_{g}\right|+\left|S \cap A_{g}\right|} \prod_{r=1}^{k} \mathbb{E}\left(f(X)^{\left|R \cap A_{r}\right|+\left|S \cap A_{r}\right|} \mid \omega\right) .
$$

By (3.2),

$$
\mathbb{E}\left(f(\sigma)^{R} f(\sigma)^{S} \mid \omega\right) \geq g_{R}(\omega) g_{S}(\omega) .
$$

By the FKG property of $\phi[18$, Theorem 3.8],

$$
\begin{aligned}
\left\langle f(\sigma)^{R} f(\sigma)^{S}\right\rangle & =\phi\left(\mathbb{E}\left(f(\sigma)^{R} f(\sigma)^{S} \mid \omega\right)\right) \\
& \geq\left\langle f(\sigma)^{R}\right\rangle\left\langle f(\sigma)^{S}\right\rangle,
\end{aligned}
$$

as required.

When $h \equiv 0$, the terms in $f(0)$ do not appear in the above, and it therefore suffices that $f \in \mathcal{F}_{q}$.

\section{Proof of Theorem 3.6}

We shall use the elementary fact that, if $T$ is a nonnegative random variable,

$$
\mathbb{E}\left(T^{m+n}\right) \geq \mathbb{E}\left(T^{m}\right) \mathbb{E}\left(T^{n}\right), \quad m, n \geq 0 .
$$


This trivial inequality may be proved in several ways, one of which is the following. Let $T_{1}$ and $T_{2}$ be independent copies of $T$. Clearly,

$$
\left(T_{1}^{m}-T_{2}^{m}\right)\left(T_{1}^{n}-T_{2}^{n}\right) \geq 0
$$

since either $0 \leq T_{1} \leq T_{2}$ or $0 \leq T_{2} \leq T_{1}$. Inequality (5.1) follows by multiplying out (5.2) and averaging.

(a) Inequality (3.3) with $I=0$ is a triviality. Since $f(X)$ is real-valued, with the same distribution as $-f(X), \mathbb{E}\left(f(X)^{m}\right)=0$ when $m$ is odd and is positive when $m$ is even. When $m+n$ is even, (3.2) follows from (5.1) with $T=f(X)^{2}$, and both sides of (3.2) are 0 otherwise.

(b) An easy calculation shows that

$$
\mathbb{E}\left(f(X)^{m}\right)= \begin{cases}1 & \text { if } q \text { divides } m \\ 0 & \text { otherwise }\end{cases}
$$

and (3.1) and (3.2) follow.

(c) Inequality (3.2) follows by (5.1) with $T=f(X)$.

\section{Proof of Theorem 3.7}

We may as well assume that $f_{0} \not \equiv 0$ so that $f_{0}(0)>0$ and $f_{1}(0)=0$. We use the notation of Section 4 , and let $F_{i}: \Omega \rightarrow \mathbb{C}$ be given by

$$
\begin{aligned}
& F_{0}(\omega)=f_{0}(0)^{\left|R \cap A_{g}\right|} \prod_{r=1}^{k} \mathbb{E}\left(f_{0}(X)^{\left|R \cap A_{r}\right|} \mid \omega\right), \\
& F_{1}(\omega)=\prod_{r=1}^{k} \mathbb{E}\left(f_{1}(X)^{\left|S \cap A_{r}\right|} \mid \omega\right) .
\end{aligned}
$$

By (3.1), $F_{0}$ and $F_{1}$ are real-valued and nonnegative. Since $f_{0} \in \mathcal{F}_{q}^{0}, F_{0}$ is nondecreasing (as in Section 4).

Since $f_{0} f_{1} \equiv 0$,

$$
\mathbb{E}\left(f_{0}(\sigma)^{R} f_{1}(\sigma)^{S} \mid \omega\right)=1_{Z}(\omega) F_{0}(\omega) F_{1}(\omega),
$$

where $1_{Z}$ is the indicator function of the event $Z=\{S \leftrightarrow R \cup\{g\}\}$. Here, as usual, we write $A \leftrightarrow B$ if there exists an open path in $\omega$ from some vertex of $A$ to some vertex of $B$. Let $T$ be the subset of $V^{+}$containing all vertices joined to $S$ by open paths, and write $\omega_{T}$ for the configuration $\omega$ restricted to $T$. Using conditional expectation,

$$
\begin{aligned}
\left\langle f_{0}(\sigma)^{R} f_{1}(\sigma)^{S}\right\rangle & =\phi\left(1_{Z} F_{0} F_{1}\right) \\
& =\phi\left(1_{Z} F_{1} \phi\left(F_{0} \mid T, \omega_{T}\right)\right),
\end{aligned}
$$


where we have used the fact that $1_{Z}$ and $F_{1}$ are functions of the pair $T, \omega_{T}$ only. On the event $Z, F_{0}$ is a nondecreasing function of the configuration restricted to $V^{+} \backslash T$. Furthermore, given $T$, the conditional measure on $V^{+} \backslash T$ is the corresponding random-cluster measure. It follows that

$$
\phi\left(F_{0} \mid T, \omega_{T}\right) \leq \phi\left(F_{0}\right) \quad \text { on the event } Z
$$

by [18, Theorem 3.21]. By (6.3),

$$
\begin{aligned}
\left\langle f_{0}(\sigma)^{R} f_{1}(\sigma)^{S}\right\rangle & \leq \phi\left(1_{Z} F_{1} \phi\left(F_{0}\right)\right) \\
& \leq \phi\left(F_{0}\right) \phi\left(F_{1}\right) \\
& =\left\langle f_{0}(\sigma)^{R}\right\rangle\left\langle f_{1}(\sigma)^{S}\right\rangle,
\end{aligned}
$$

and the theorem is proved.

When $h \equiv 0, A_{g}=\{g\}$ in (6.1), and it suffices that $f_{0} \in \mathcal{F}_{q}$.

\section{Acknowledgements}

This work was supported in part by the Engineering and Physical Sciences Research Council under grant EP/I03372X/1. The author is grateful to Jakob Björnberg for proposing the model with angular spins and also to Chuck Newman and Aernout van Enter for their comments and suggestions.

\section{References}

[1] M. Aizenman, "Geometric analysis of $\varphi^{4}$ fields and Ising models, I-II", Comm. Math. Phys. 86:1 (1982), 1-48.

[2] M. Aizenman and B. Nachtergaele, "Geometric aspects of quantum spin states", Comm. Math. Phys. 164:1 (1994), 17-63.

[3] J. E. Björnberg, "Vanishing critical magnetization in the quantum Ising model", Comm. Math. Phys. 337:2 (2015), 879-907.

[4] J. E. Björnberg and G. R. Grimmett, "The phase transition of the quantum Ising model is sharp", J. Stat. Phys. 136:2 (2009), 231-273.

[5] N. Crawford and D. Ioffe, "Random current representation for transverse field Ising model", Comm. Math. Phys. 296:2 (2010), 447-474.

[6] J. De Coninck, A. Messager, S. Miracle-Sole, and J. Ruiz, "A study of perfect wetting for Potts and Blume-Capel models with correlation inequalities", J. Statist. Phys. 52:1-2 (1988), 45-60.

[7] R. Fernández, J. Fröhlich, and A. D. Sokal, Random walks, critical phenomena, and triviality in quantum field theory, Springer, Berlin, 1992.

[8] C. M. Fortuin, "On the random-cluster model, II: The percolation model”, Physica 58:3 (1972), 393-418.

[9] C. M. Fortuin, "On the random-cluster model, III: The simple random-cluster model", Physica 59:4 (1972), 545-570.

[10] C. M. Fortuin and P. W. Kasteleyn, "On the random-cluster model, I: Introduction and relation to other models", Physica 57:4 (1972), 536-564. 
[11] C. M. Fortuin, P. W. Kasteleyn, and J. Ginibre, "Correlation inequalities on some partially ordered sets”, Comm. Math. Phys. 22:2 (1971), 89-103.

[12] N. Ganikhodjaev and F. A. Razak, "Griffith-Kelly-Sherman correlation inequalities for generalized Potts model", Math. Phys. Anal. Geom. 13:1 (2010), 1-18.

[13] J. Ginibre, "General formulation of Griffiths' inequalities", Comm. Math. Phys. 16:4 (1970), 310-328.

[14] R. B. Griffiths, "Correlations in Ising ferromagnets, I", J. Math. Phys. 8:3 (1967), 478-483.

[15] R. B. Griffiths, "Correlations in Ising ferromagnets, II: External magnetic fields", J. Math. Phys. 8:3 (1967), 484-489.

[16] R. B. Griffiths, "Rigorous results for Ising ferromagnets of arbitrary spin", J. Math. Phys. 10:9 (1969), 1559-1565.

[17] R. B. Griffiths, C. A. Hurst, and S. Sherman, "Concavity of magnetization of an Ising ferromagnet in a positive external field", J. Math. Phys. 11:3 (1970), 790-795.

[18] G. R. Grimmett, The random-cluster model, Grundlehren der math. Wissenschaften 333, Springer, Berlin, 2006.

[19] G. R. Grimmett, "Space-time percolation”, pp. 305-320 in In and out of equilibrium, 2 (Rio de Janeiro, 2006), edited by V. Sidoravicius and M. E. Vares, Progr. Probab. 60, Birkhäuser, Basel, 2008.

[20] D. G. Kelly and S. Sherman, "General Griffiths' inequalities on correlations in Ising ferromagnets”, J. Math. Phys. 9:3 (1968), 466-484.

[21] E. H. Lieb, “A refinement of Simon's correlation inequality”, Comm. Math. Phys. 77:2 (1980), $127-135$.

[22] B. M. McCoy and T. T. Wu, The two-dimensional Ising model, Harvard University, Cambridge, MA, 1973.

[23] R. H. Schonmann, "On two correlation inequalities for Potts models", J. Statist. Phys. 52:1-2 (1988), 61-67.

[24] B. Simon, "Correlation inequalities and the decay of correlations in ferromagnets", Comm. Math. Phys. 77:2 (1980), 111-126.

Received 3 Dec 2015. Accepted 28 Mar 2016.

GEOFFREY R. GRIMMETT: g.r.grimmett@statslab.cam.ac.uk

Statistical Laboratory, Centre for Mathematical Sciences, University of Cambridge, Cambridge, CB3 OWB, United Kingdom 
EDITORIAL BOARD

ANTONIO CARCATERRA

ERIC A. CARLEN

FRANCESCO DELL'ISOLA

RAFFAELE ESPOSITO

ALBERT FANNJIANG

Gilles A. FranCFORT

Pierangelo MARCATI

JEAN-JACQUES MARIGO

PETER A. MARKOWICH

MARTIN OSTOJA-STARZEWSKI

PIERRE SEPPECHER

DAVID J. STEIGMANN

PAUl STEINMANN

PierRe M. SuQueT

MANAGING EDITORS

MICOL AMAR

CORRADO LATTANZIO

ANGELA MADEO

MARTIN OSTOJA-STARZEWSKI

ADVISORY BOARD

ADNAN AKAY

Holm AltenBaCH

MICOL AMAR

HARM ASKES

TEODOR ATANACKOVIĆ

VICTOR BERDICHEVSKY

GUY BOUCHITTÉ

ANDREA BRAIDES

ROBERTO CAMASSA

MAURO CARFORE

ERIC DARVE

FELIX DARVE

ANNA DE MASI

GianPiEtro DEL Piero

EMMANUELE Di BENEDETTO

BERNOLD FIEDLER

IRENE M. GAMBA

DAVID Y. GAO

SERGEY GAVRILYUK

TIMOTHY J. HEALEY

DOMINIQUE JEULIN

ROGER E. KHAYAT

CORRADO LATTANZIO

ROBERT P. LIPTON

ANGELO LUONGO

ANGELA MADEO

JUAN J. MANFREDI

CARLO MARCHIORO

GÉRARD A. MAUGIN

ROBERTO NATALINI PATRIZIO NEFF

ANDREY PIATNITSKI

ERRICO PRESUTTI

MARIO PULVIRENTI

LUCIO RUSSO

Miguel A. F. SANJUAN

PATRICK SElVADURAI

ALEXANDER P. SEYRANIAN

MIROSLAV ŠILHAVÝ

GUIDO SWEERS

ANTOINETTE TORDESILLAS

LEV TRUSKINOVSKY

JUAN J. L. VELÁZQUEZ VINCENZO VESPRI ANGELO VULPIANI msp.org/memocs

Università di Roma "La Sapienza", Italia

Rutgers University, USA

(CO-CHAIR) Università di Roma "La Sapienza", Italia

(TREASURER) Università dell'Aquila, Italia

University of California at Davis, USA

(CO-CHAIR) Université Paris-Nord, France

Università dell'Aquila, Italy

École Polytechnique, France

DAMTP Cambridge, UK, and University of Vienna, Austria

(CHAIR MANAGING EDITOR) Univ. of Illinois at Urbana-Champaign, USA

Université du Sud Toulon-Var, France

University of California at Berkeley, USA

Universität Erlangen-Nürnberg, Germany

LMA CNRS Marseille, France

Università di Roma "La Sapienza", Italia

Università dell'Aquila, Italy

Université de Lyon-INSA (Institut National des Sciences Appliquées), France

(CHAIR MANAGING EDITOR) Univ. of Illinois at Urbana-Champaign, USA

Carnegie Mellon University, USA, and Bilkent University, Turkey

Otto-von-Guericke-Universität Magdeburg, Germany

Università di Roma "La Sapienza", Italia

University of Sheffield, UK

University of Novi Sad, Serbia

Wayne State University, USA

Université du Sud Toulon-Var, France

Università di Roma Tor Vergata, Italia

University of North Carolina at Chapel Hill, USA

Università di Pavia, Italia

Stanford University, USA

Institut Polytechnique de Grenoble, France

Università dell'Aquila, Italia

Università di Ferrara and International Research Center MEMOCS, Italia

Vanderbilt University, USA

Freie Universität Berlin, Germany

University of Texas at Austin, USA

Federation University and Australian National University, Australia

Université Aix-Marseille, France

Cornell University, USA

École des Mines, France

University of Western Ontario, Canada

Università dell' Aquila, Italy

Louisiana State University, USA

Università dell'Aquila, Italia

Université de Lyon-INSA (Institut National des Sciences Appliquées), France University of Pittsburgh, USA

Università di Roma "La Sapienza”, Italia

Université Paris VI, France

Istituto per le Applicazioni del Calcolo "M. Picone", Italy

Universität Duisburg-Essen, Germany

Narvik University College, Norway, Russia

Università di Roma Tor Vergata, Italy

Università di Roma "La Sapienza”, Italia

Università di Roma “Tor Vergata”, Italia

Universidad Rey Juan Carlos, Madrid, Spain

McGill University, Canada

Moscow State Lomonosov University, Russia

Academy of Sciences of the Czech Republic

Universität zu Köln, Germany

University of Melbourne, Australia

École Polytechnique, France

Bonn University, Germany

Università di Firenze, Italia

Università di Roma La Sapienza, Italia

MEMOCS (ISSN 2325-3444 electronic, 2326-7186 printed) is a journal of the International Research Center for the Mathematics and Mechanics of Complex Systems at the Università dell'Aquila, Italy.

Cover image: "Tangle” by $\odot$ John Horigan; produced using the Context Free program (contextfreeart.org).

PUBLISHED BY

7 mathematical sciences publishers

nonprofit scientific publishing

http://msp.org/

(C) 2016 Mathematical Sciences Publishers 
Special issue in honor of

Lucio Russo

Lucio Russo: A multifaceted life

Raffaele Esposito and Francesco dell'Isola

The work of Lucio Russo on percolation Geoffrey R. Grimmett

"Mathematics" and "physics" in the science of harmonics Stefano Isola

From quantum to classical world: emergence of trajectories in a quantum system Rodolfo Figari and Alessandro Teta

Propagation of chaos and effective equations in kinetic theory: a brief survey Mario Pulvirenti and Sergio Simonella

What decides the direction of a current? Christian Maes

A remark on eigenvalue perturbation theory at vanishing isolation distance Fiorella Barone and Sandro Graffi

Some results on the asymptotic behavior of finite connection probabilities in percolation Massimo Campanino and Michele Gianfelice

Correlation inequalities for the Potts model Geoffrey R. Grimmett

Quantum mechanics: some basic techniques for some basic models, I: The models Vincenzo Grecchi

Quantum mechanics: some basic techniques for some basic models, II: The techniques Vincenzo Grecchi

On stochastic distributions and currents

Vincenzo Capasso and Franco Flandoli

A note on Gibbs and Markov random fields with constraints and their moments Alberto Gandolfi and Pietro Lenarda

Quantum mechanics: light and shadows (ontological problems and epistemic solutions) Gianfausto Dell'Antonio

MEMOCS is a journal of the International Research Center for the Mathematics and Mechanics of Complex Systems at the Università dell' Aquila, Italy.

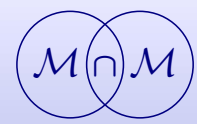

